\title{
Ictioplancton superficial de la cuenca del océano Pacífico colombiano (septiembre 2003)*
}

\author{
Eugenia Escarria $^{1}$, Beatriz S. Beltrán-León ${ }^{2}$ \& Alan Giraldo ${ }^{1}$ \\ ${ }^{1}$ Universidad del Valle, Departamento de Biología \\ Grupo de Investigación en Ecología Animal, A.A. 25360 Cali, Colombia \\ ${ }^{2}$ Ministerio de Ambiente, Vivienda y Desarrollo Territorial, Unidad Administrativa Especial del \\ Sistema de Parques Nacionales Naturales, Dirección Territorial Sur Occidente, Cali, Colombia
}

\begin{abstract}
RESUMEN. Se presentan los resultados del análisis taxonómico y ecológico del ictioplancton obtenido mediante arrastres superficiales en 23 estaciones de muestreo durante la campaña oceanográfica Pacífico XXXVIII ERFEN-XXXVI en la cuenca del Océano Pacífico colombiano. Se identificaron estadios larvales de 19 especies pertenecientes a 18 familias, en su mayoría de interés para la pesca. Las familias más abundantes fueron Photichthyidae (179 larvas·1000 m³ y Hemiramphidae (106 larvas·1000 $\mathrm{m}^{-3}$ ). Se determinó la distribución y abundancia de huevos mediante el análisis gráfico de mapas de dispersión espacial. Destacaron por su frecuencia de ocurrencia y abundancia las larvas de Vinciguerria lucetia (Familia Photichthydae) con 112 larvas $1000 \mathrm{~m}^{-3}$, Cetengraulis mysticetus (Familia Engraulidae) con 104 larvas 1000 $\mathrm{m}^{-3}$, y Hyporhamphus sp. y Oxyporhamphus sp. (Familia Hemiramphidae) con 73 y 33 larvas $1000 \mathrm{~m}^{-3}$ respectivamente. Considerando las limitaciones del muestreo (hora, tipo de red y arrastre), es muy probable que los valores de abundancia de larvas fueron subestimados.
\end{abstract}

Palabras clave: ictioplancton, huevos de peces, larvas de peces, Colombia, Océano Pacífico.

\section{Surface ichthyoplankton in the Pacific Ocean off Colombia (September 2003)*}

\begin{abstract}
We show the results of taxonomic and ecological analyses carried out on ichthyoplankton caught in 23 surface tows during the PACIFICO XXXVIII-ERFEN XXXVI oceanographic research campaign in the Colombian Pacific Ocean. Larvae of 19 fish species belonging to 18 families were identified. Most of the fish larvae were commercial value species. The most abundant family was Photichthyidae (179 larvae $1000 \mathrm{~m}^{-3}$ ), followed by Hemiramphidae with 106 larvae $1000 \mathrm{~m}^{-3}$. The spatial distribution and abundance of fish larvae and eggs were determined using dispersion maps (graphic analysis). Fish larvae abundances were probably underestimated due to sampling limitations (hour, type of net, and surface towing). However, larval abundances of Vinciguerria lucetia (Family Photichthydae: 112 larvae·1000 $\mathrm{m}^{-3}$ ), Cetengraulis mysticetus (Family Engraulidae: 104 larvae $1000 \mathrm{~m}^{-3}$ ), Hyporhamphus sp. (Family Hemiramphidae: 73 larvae $1000 \mathrm{~m}^{-3}$ ), and Oxyporhamphus sp. (Family Hemiramphidae: 33 larvae $1000 \mathrm{~m}^{-3}$ ) were outstanding.
\end{abstract}

Key words: Ichthyoplankton, fish eggs, fish larvae, Colombia, Pacific Ocean.

Autor corresponsal: Alan Giraldo (ecologia@univalle.edu.co)

El ictioplancton constituye un componente importante en las comunidades planctónicas y comprende los huevos, larvas, postlarvas y, algunas veces, los juveniles de peces (Ciechomski, 1981; Matsuura \& Olivar, 1999). Su relevancia radica en que proporcio- na el conocimiento necesario para comprender aspectos de las pesquerías encaminados hacia el manejo racional. En este sentido, los stocks de peces y sus potenciales de explotación son evaluados mediante investigaciones sistemáticas que requieren el estudio

* Trabajo presentado en el XXV Congreso de Ciencias del Mar de Chile y XI Congreso Latinoamericano de Ciencias del Mar (COLACMAR), realizados en Viña del Mar, entre el 16 y 20 de mayo de 2005. 
de huevos y larvas (Rueda-Montenegro \& Beltrán, 1992; Beltrán-León \& Ríos, 2000). Estos estudios sirven como base para la determinación de zonas y épocas de desove, biomasa de adultos, efectivos desovantes, migración de poblaciones y abundancia de larvas y adultos. El estudio del ictioplancton en el Pacífico colombiano comenzó con los trabajos de Echeverri \& Bergamín (1982), López-Peralta (1984) y Rueda-Montenegro \& Caraballo (1984). Entre los estudios más recientes y completos se encuentra el de Beltrán-León \& Ríos (2000), quienes realizaron el análisis taxonómico de larvas y postlarvas, y el de Escobar (2004), que muestra la relación entre la abundancia del ictioplancton durante el fenómeno El Niño, ambos en el Pacífico colombiano.

Con este trabajo se pretende contribuir al conocimiento de la composición, distribución y abundancia del ictioplancton superficial en la cuenca del Pacífico colombiano $\left(1^{\circ} 30^{\prime}-6^{\circ} 30^{\prime} \mathrm{N}, 77^{\circ} 45^{\prime}-84^{\circ} 00^{\prime} \mathrm{W}\right)$, con el fin de determinar posibles áreas de desove y alevinaje. El estudio se realizó en el marco de la campaña oceanográfica PACÍFICO XXXVIII - ERFEN XXXVI, con el buque oceanográfico ARC "Malpelo" entre el 1 y 25 de septiembre de 2003. Se utilizó una red cónica simple de 363 micras de abertura de malla, provista de un flujómetro digital General Oceanics, para realizar pescas superficiales de zooplancton en 23 estaciones de muestreo, durante 10 min a 3 nudos. Considerando la amplitud de la plataforma continental en el área de estudio, las estaciones se agruparon en costeras (separadas entre sí por $30 \mathrm{mn}$ ) y oceánicas (separadas entre sí por $60 \mathrm{mn}$ ). Las muestras se preservaron en formalina neutralizada al $10 \%$ en agua de mar y se identificaron larvas y huevos sobre la base de los caracteres merísticos, morfométricos y de pigmentación, siguiendo la clave de Beltrán-León \& Ríos (2000). Se registró la abundancia de cada especie (ind. $1000 \mathrm{~m}^{-3}$ ) y se estimó el biovolumen de zooplancton $(\mathrm{mL} \cdot 1000$ $\mathrm{m}^{-3}$ ). Ambos se representaron en mapas de distribución utilizando los rangos de abundancia utilizados por Beltrán-León \& Ríos (1992).

El biovolumen osciló entre 26,12 y 661,57 $\mathrm{mL} \cdot 1000 \mathrm{~m}^{-3}$, con un promedio de $232,76 \mathrm{~mL} \cdot 1000$ $\mathrm{m}^{-3}$. Se consideró la época de estudio como de producción media, puesto que registró en su mayoría valores medios de biomasa (Fig. 1a). Todas las estaciones fueron positivas para huevos de peces y se contabilizó un total de 24.408 huevos $1000 \mathrm{~m}^{-3}$. La abundancia fluctuó entre 8 y 5.687 huevos $1000 \mathrm{~m}^{-3}$, registrándose los valores más bajos en las estaciones costeras 1,3 y 7 (Fig. 1b).

La abundancia de larvas varió entre 7 y 200 larvas. $1000 \mathrm{~m}^{-3}$. Se identificaron los estadios larvales de 19 especies pertenecientes a 18 familias (Tabla 1). Vinciguerria lucetia (Photichthydae, 112 larvas $\cdot 1000 \mathrm{~m}^{-3}$ ) fue la más abundante (18\%). Sin
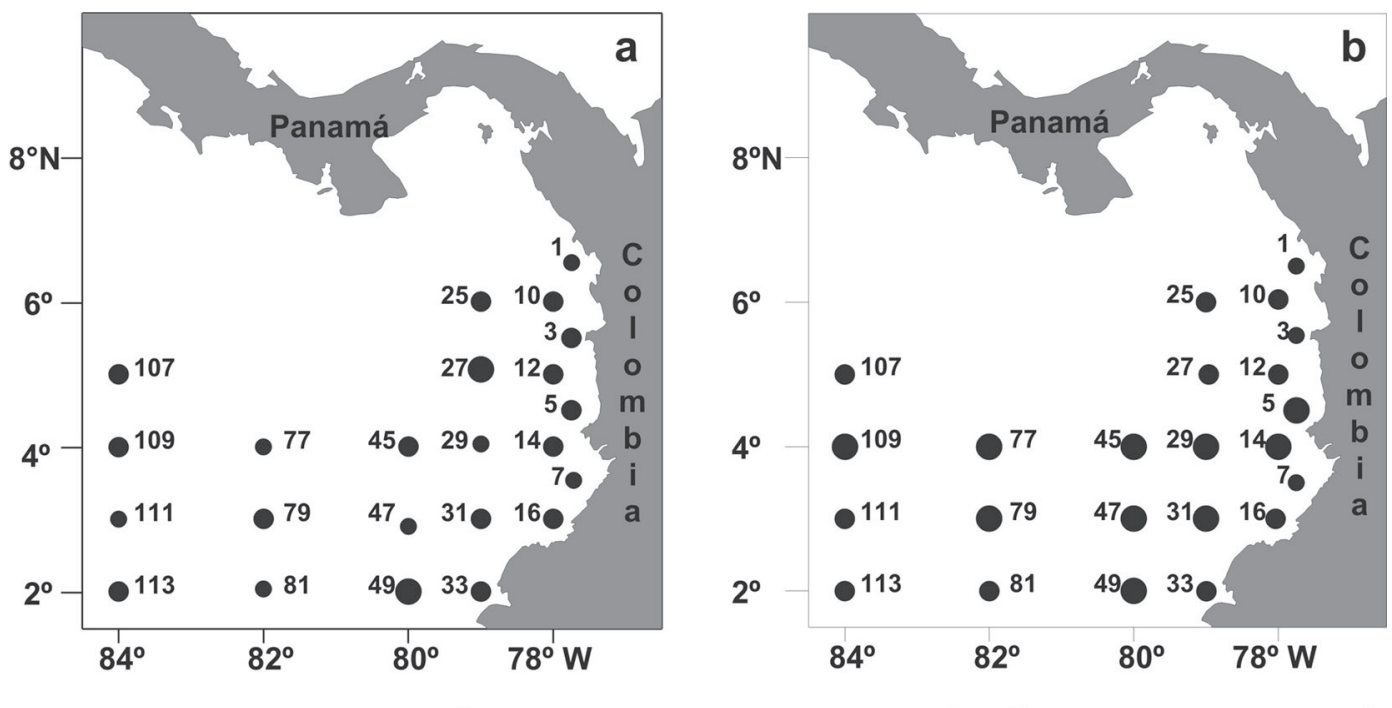

- Baja: 1 - $99 \mathrm{~mL} \cdot 1000 \mathrm{~m}^{-3}$

Media: $100-499 \mathrm{~mL} \cdot 1000 \mathrm{~m}^{-3}$

Alta: $>500 \mathrm{~mL} \cdot 1000 \mathrm{~m}^{-3}$

Figura 1. Distribución espacial de a) biovolumen de zooplancton, y b) huevos de peces en la cuenca del Océano Pacífico colombiano, en septiembre de 2003.

Figure 1. Spatial distribution of a) zooplankton biovolume, and b) fish eggs in the Colombian Pacific Ocean in September 2003. 
embargo, la familia Hemiramphidae (Hyporhamphus sp. y Oxyporhamphus sp.) fue la de más amplia distribución (Fig. 2a). Destacó por su abundancia y frecuencia Cyclothone acclinidens (Gonostomatidae: 74 larvas $1000 \mathrm{~m}^{-3}$ ), pocas veces detectada en esta zona (Fig. 2b).

La abundancia relativa de la familia Engraulidae (Cetengraulis mysticetus) fue de 12\%; mientras que las especies pertenecientes a la familia Mycthophidae (Diogenichthys laternatus, Myctophum aurolaternatum, Lampanyctus sp. y Gonichtys sp.) fue de 7\%, con una distribución espacial aleatoria. La familia Mugilidae (Mugil cephalus) se encontró en las estaciones costeras desde bahía Solano hasta el sur de la bahía de Buenaventura, mientras que las especies de la familia Carangidae (Chloroscombrus orqueta,
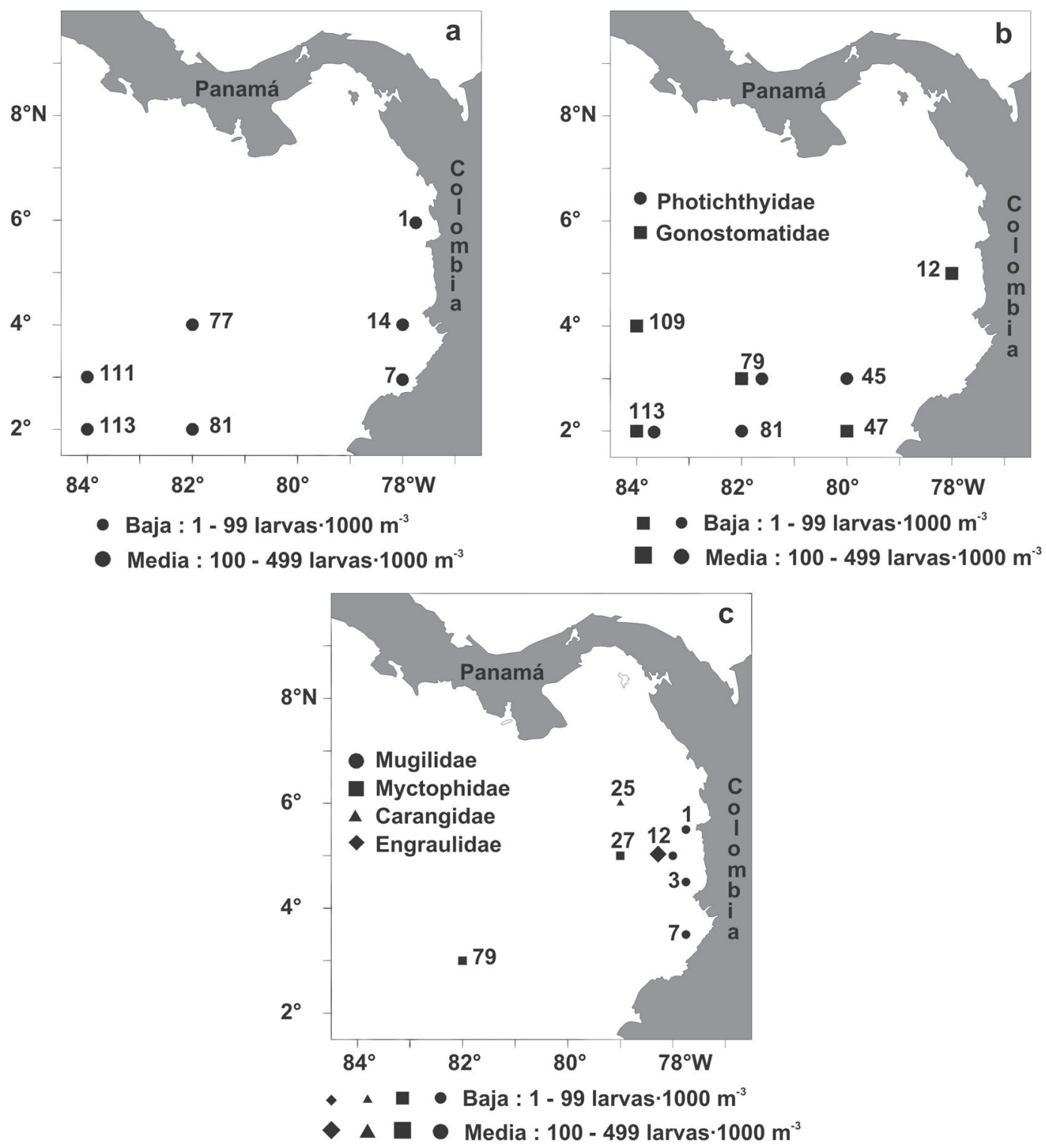

Figura 2. Distribución espacial de las familias de larvas de peces en la cuenca del oceáno Pacífico colombiano en septiembre de 2003. a) Hemirramphidae, b) Photichthyidae y Gonostomatidae, c) Mugilidae, Myctophidae, Engraulidae y Carangidae.

Figure 2. Spatial distibution of fish larvae families in the Colombian Pacific Ocean in september 2003. a) Hemirramphidae, b) Photichthyidae and Gonostomatidae, c) Mugilidae, Myctophidae, Engraulidae and Carangidae. 
Tabla 1. Registro taxonómico del ictioplancton superficial de la cuenca del Pacífico colombiano en septiembre 2003.

Table 1. Taxonomic classification of surface icthyoplankton captured in the Pacific Ocean of Colombia in september 2003.

\begin{tabular}{|c|c|c|}
\hline Familia/especie & $\begin{array}{l}\text { Abundancia promedio } \\
\left.\text { (larvas } \cdot 1000 \mathrm{~m}^{-3}\right)\end{array}$ & $\begin{array}{c}\text { Composición } \\
\text { por familia (\%) }\end{array}$ \\
\hline Hemiramphidae & & 15 \\
\hline Hyporhamphus sp. & 73 & \\
\hline Oxyporhamphus sp. & 33 & \\
\hline Photichthydae & & 18 \\
\hline Vinciguerria lucetia & 112 & \\
\hline Gonostomatidae & & 11 \\
\hline Cyclothone acclinidens & 74 & \\
\hline Engraulidae & & 12 \\
\hline Cetengraulis mysticetus & 104 & \\
\hline Mycthophidae & & 7 \\
\hline Diogenichthys laternatus & 12,5 & \\
\hline Myctophum aurolaternatum & 12,5 & \\
\hline Lampanyctus sp. & 28 & \\
\hline Gonichtys sp. & 28 & \\
\hline Mugilidae & & 6 \\
\hline Mugil cephalus & 56 & \\
\hline Carangidae & & 4 \\
\hline Chloroscombrus orqueta & 11,5 & \\
\hline Caranx sp. & 11,5 & \\
\hline C. caballus & 11,5 & \\
\hline Decapterus sp. & 11,5 & \\
\hline Scombridae & 19 & 2 \\
\hline Pomacentridae & 32 & 2 \\
\hline Gobiidae & 13 & 1 \\
\hline Paralichthyidae & & 1 \\
\hline Citharichthys platophrys & 9 & \\
\hline Cynoglossidae & & 1 \\
\hline Symphurus sp. & 9 & \\
\hline Coryphaenidae & & 1 \\
\hline Coryphaena hippurus & 9 & \\
\hline Melanostomidae & & 1 \\
\hline Bathophilus filifer & 24 & \\
\hline Scorpaenidae & 9 & 1 \\
\hline Gempylidae & 11 & 1 \\
\hline Oneirodidae & & 1 \\
\hline Oneirodes spp. & 25 & \\
\hline Melamphidae & & 1 \\
\hline Melamphaes sp. & 12 & \\
\hline Stomiidae & & 1 \\
\hline Bathophilus filifer & & \\
\hline
\end{tabular}


Caranx sp., C. caballus y Decapterus sp.) solo se obtuvieron en una estación costera, representando el 4\% del total (Fig. 2c).

Los valores de bivolumen registrados en septiembre pueden ser considerados como valores medios según los rangos establecidos por Beltrán-León \& Ríos (2000). Las concentraciones más altas se registraron en la zona costera, probablemente influenciadas por la topografía del fondo (costa somera), la abundancia de alimento (biomasa fitoplanctónica) y las condiciones geomorfológicas locales (cabos, bahías y estuarios).

Debido a que los huevos de peces son derivadores pasivos es de esperar alta frecuencia de los mismos en la parte oceánica influenciados por el patrón de circulación superficial, el cual se ve afectado por la corriente de Colombia que en el mes analizado (septiembre) es más intensa (vientos alisios del sur hacia el noroeste). Esto implica necesariamente el transporte de huevos fuera de la costa, aumentando su densidad en la parte oceánica (i.e. López-Peralta, 1984). Aunque la presencia de larvas estuvo estrechamente asociada con la de huevos, su abundancia fue baja llegando incluso a no colectarse larvas en algunas estaciones costeras. Además, familias de amplia distribución oceánica, como Myctophidae no fueron abundantes, debido probablemente a la hora del muestreo (generalmente diurno), ya que estos organismos realizan migraciones verticales diarias. Además, el tipo de red utilizado (cónica simple) no fue el más adecuado para estos estudios, ya que el armazón de la red favorece la evasión de las larvas. Por lo tanto, es necesario efectuar arrastres oblicuos con redes Bongo para cubrir toda la columna de agua. Los resultados obtenidos son consistentes con los registrados por Escobar (2004) en esta misma área, para tres períodos distintos. En este sentido, las larvas registradas han sido previamente reportadas en diferentes estadios de desarrollo (López-Peralta, 1984; Beltrán-León \& Ríos, 2000).

Debido a la baja resolución espacial y temporal del muestreo, no fue posible establecer alguna tendencia de agregación para definir áreas y/o épocas de desove o reclutamiento de peces. A pesar que el tipo de aproximación metodológica utilizada en este estudio no permite evaluar la biomasa esperada de poblaciones de peces futuras, la información derivada incrementa el conocimiento sobre el ictioplancton en esta región.

\section{REFERENCIAS}

Beltrán-León, B.S. \& R. Ríos. 2000. Estadios tempranos de peces del Pacífico colombiano. Instituto Nacional de Pesca y Acuicultura INPA-Buenaventura, $727 \mathrm{pp}$.

Ciechomski, J. 1981. Ictioplancton. En: D. Boltovskoy (ed.). Atlas del zooplancton del Atlántico sudoccidental y métodos de trabajo con zooplancton marino. Instituto de Investigación y Desarrollo Pesquero, Publicación Especial, Mar de Plata, pp. 829-861.

Echeverri, C. \& H. Bergamín. 1982. Estudio preliminar de la taxonomía y distribución de larvas de peces en la bahía de Buenaventura. Tesis de Biología, Universidad del Valle, Cali, 99 pp.

Escobar, N. 2004. Variación del ictioplancton en la cuenca del Pacífico colombiano durante los periodos 23. jun.-12. jul. 01, 27 ago.-15. sep. 01 y 03-22. sep. 02. Estudio Regional del Fenómeno del Niño (ERFEN). Trabajo de Grado. Facultad de Ciencias, Universidad Militar Nueva Granada, Bogotá, 88 pp.

López-Peralta, R.H. 1984. Descripción y abundancia del ictioplancton entre 0 y $10 \mathrm{~m}$ de profundidad para el Pacífico colombiano (Nov. 1982). Tesis de Biología Marina. Universidad Jorge Tadeo Lozano. Facultad de Biología Marina, Bogotá, 218 pp.

Matsuura, Y. \& M.P. Olivar. 1999. Fish larvae. En: D. Boltovskoy (ed.). South Atlantic zooplankton. Backhuys Publishers, Leiden, pp. 1445-1496.

Rueda-Montenegro, C. \& P. Caraballo. 1984. Composición, distribución y abundancia del ictioneuston en el Pacífico colombiano en noviembre y diciembre de 1982. Tesis de Biología Marina, Universidad Jorge Tadeo Lozano, Bogotá, 199 pp.

Rueda-Montenegro, C. \& B. Beltrán. 1992. Ictioplancton de las familias Clupeidae y Engraulidae en el litoral del Pacífico colombiano, durante 1991. Memorias del VIII Seminario Nacional de Ciencias y Tecnología del Mar y Congreso Centroamericano y del Caribe de Ciencias del Mar. Santa Marta, 2: 735-743. 